\title{
ESTHÉTIQUE DE LA TECHNIQUE
}

\author{
Sophie A. de Beaune et Liliane Hilaire-Pérez
}

\begin{abstract}
A ristote se posait déjà la question des rapports entre le beau, l'utile et le nécessaire : la beauté a-t-elle à voir avec la technique ? La technique est-elle forcément laide ? La beauté est-elle nécessairement inutile ? Il reprenait là le dialogue engagé à ce sujet dans le Grand Hippias de Platon. Socrate y pose tour à tour la plupart des questions qui agitent les théoriciens de l'art jusqu'à aujourd'hui : peut-on dire que « ce qui sied est plus beau que ce qui ne sied pas »? Est-ce que « ce qui pour nous est beau [serait] ce qui éventuellement est utilisable »?

Cette question, une tradition philosophique qui court depuis l'Antiquité, et qu'on voit s'exprimer encore chez Kant, Hegel ou Heidegger, l'a tranchée en dissociant nettement esthétique et technique. Une dissociation qui ne va cependant pas toujours de soi. Pendant des siècles, la richesse sémantique du terme " art » et le rôle unificateur qu'il a joué dans l'espace académique et savant à l'époque moderne a porté des innovations, telles les sociétés des arts, animée d'un idéal de conception unitaire des objets et d'un rêve d'harmonie entre les sciences et les arts, unis au nom d'une compréhension synthétique de l'invention - et finalement de tout acte opératoire - comme relevant d'un art des liaisons selon Diderot ${ }^{1}$. Insistons sur cet art des rapports. Au milieu du siècle, William Hogarth conçoit le plaisir esthétique comme goût des correspondances ("exactness of counterparts"). Dans les années 1790, Joshua Reynolds, chantre de la beauté néo-classique, définit l'originalité comme la combinaison de modèles connus ${ }^{2}$. Entre-temps, comme on le reprécisera, Adam Smith, dans l'Essai sur la nature de l'imitation dans les arts, voit dans " l'écart entre l'objet qui imite et l'objet imité le fondement de la beauté de l'imitation » et de l'art, érigeant la « maitrise de la disparité » en

* Sophie Archambault de Beaune est professeur à l'université Lyon 3 et chercheur à l'UMR 7041 "Archéologies et sciences de l'Antiquité ». Ses recherches portent sur les comportements techniques et les aptitudes cognitives de l'homme préhistorique. Elle a notamment publié L'Homme et l'outil (Paris, CNRS Éditions, 2008). Adresse : UMR 7041 ArScAn, 21, allée de l'université, F-92023 Nanterre cedex (sophie.de-beaune@mae.cnrs.fr).

Liliane Hilaire-Pérez est professeur à l'université Paris 7 (laboratoire « Identités, cultures, territoires ", EA 337) et directrice d'études à l'EHESS (Centre Alexandre-Koyré). Ses travaux concernent l'histoire de l'invention et des cultures opératoires en Europe à l'époque moderne. Elle a notamment publié L'Invention technique au siècle des Lumières (Paris, Albin Michel, 2000). Adresse : Université Paris 7, ICT, 5, rue Thomas Mann, F-75013 Paris (liliane.perez@wanadoo.fr).

1. Hilaire-PÉrez, 2002.

2. REYNOLDS, 1797.
\end{abstract}


canon artistique ${ }^{3}$. Cette conception structuraliste de la beauté fait écho au principe de réduction, comme le soulignait Didier Deleule : «L'artifice du créateur, comme celui du technicien, du savant, du philosophe, effectue le système comme œuvre de l'art : une réduction de la diversité à certains principes d'intelligibilité qui ont pour mission de respecter l'hétérogénéité tout en surmontant [...] la difficulté inhérente à la disparité des objets considérés ${ }^{4}$. » Ainsi, « tout système a valeur esthétique ${ }^{5}$ ».

Cette utopie ne résiste pas à la dissociation entre les arts et les beaux-arts à partir du $\mathrm{XVIII}^{\mathrm{e}}$ siècle. La conception du dessin - longtemps associé au dessein, à l'art du projet, à l'ingenium - en est transformée. Comme l'a montré Frédéric Morvan ${ }^{6}$, alors que l'enseignement du dessin progresse dans des écoles où se forme un milieu artisanal et technicien rôdé à la copie, à la transposition, au dimensionnement - clés de voûte d'une recomposition technologique des métiers, en termes de compétences transverses et sectorielles -, en même temps s'affirme le prestige des Beaux-Arts et d'une création artistique dégagée des conditions techniques et matérielles de production des œuvres d'art dans les ateliers ${ }^{7}$. Les historiens et les sociologues de l'art ont souligné les enjeux sociaux culturels et politiques que recouvrent cette émancipation de l'artiste, alors que les interférences avec le monde des métiers, avec le milieu des experts et des marchands étaient encore très fortes au siècle des Lumières et jusque sous la Révolution.

Un témoin de ces tensions est l'architecture. Dans De architectura, Vitruve plaçait cet art - au sens d'artifice - à l'articulation entre commodité, solidité et beauté. Mais du fait de son utilité, l'architecture a été considérée bien souvent comme un art mineur. Estelle Thibault nous fait ainsi découvrir les réflexions qui se tissent à la fin du XIX ${ }^{\mathrm{e}}$ siècle entre architectes et philosophes sur la place que l'architecture doit prendre parmi les arts : alors qu'en 1860 les membres de l'Institut, pour qui le Beau était un idéal détaché des nécessités matérielles, rejetaient hautainement l'architecture du côté de l'industriel, elle commence au début du $\mathrm{Xx}^{\mathrm{e}}$ siècle à revendiquer sans honte un statut d'art appliqué visant à une « beauté rationnelle ». C'est qu'entretemps les relations entre le beau et l'utile se sont quasiment inversées. Les travaux récents sur les expositions universelles

3. Le texte d'Adam Sмiтh, On the Nature of that Imitation which takes Place in what are called the Imitative Arts, commencé en 1777 et paru dans l'édition posthume de son œuvre établie par Dugald Stewart (1795), a été republié dans ThIerRy, dir., 1997 (ici p. 50 et 53). Le thème participe de l'esthétique de la machine et de l'œuvre d'art comme assemblage et système : voir BECQ, 1983 ; DÉmoris, 1983 ; DAMISCH, 1983 ; SCOTT, 1999.

4. Deleule, 1997, p. 31. Le verbe « surmonter » fait écho à la citation de l'abbé Jean-Baptiste Dubos en 1733, s'insurgeant contre la formalisation excessive des règles de l'art en poésie : "rien n'aide un poete françois à surmonter les difficultez, que son genie, son oreille et sa perseverance. Aucune methode reduite en art ne vient à son secours », dans Dubourg Glatigny et Vérin, 2008, p. 74.

5. On rapprochera cette formule de celle d'Hélène Vérin commentant le sens que revêt l'œuvre d'art pour Herbert Simon, et l'ancienneté de cette vision, qui place l'économie au cœur de l'esthétique : «En quelque sorte l'œuvre d'art est réussie dans la mesure où, entre les résultats et le processus qui y a conduit, on découvre une relation réussie » (VÉRIN, 1998, p. 127).

6. Morvan, 2011.

7. Voir également Millet, 2011 et les travaux à paraître dans Lembré (Stéphane) et Millet (Audrey), L'Enseignement du dessin entre art et industrie (XVIII -XIX $X^{e}$ siècles). Renouvellements historiographiques et pistes de recherche. On peut aussi faire référence à la journée d'étude organisée par Lembré et Millet, Entre art et industrie : les enjeux de la formation technique (XVIII -XIXe siècles), 11 juin 2012, IDHE, UMR-8533, Université Paris 8-Vincennes-Saint-Denis. 
mettent en valeur les revendications esthétiques - et non pas techniques - des fabricants dans bien des domaines, au nom de la qualité des produits ${ }^{8}$. Eugénie Briot, dans son analyse des mises en scène de la parfumerie aux expositions de 1889 et de 1900 , révèle que « la dimension technique des produits de parfumerie est presque entièrement absente $^{9}$ ». De même pour les papiers peints, Bernard Jacqué explique que « les papiers peints imprimés mécaniquement [...] ne retiennent l'intérêt ni des manufacturiers, ni des jurys, encore moins des publics, sinon pour les dénigrer, brièvement, jusqu'aux années 1870 », non sans nationalisme. La création d'une catégorie des beaux-arts, en 1855, a été l'occasion de rivaliser par l'exposition de " tableaux 》 (réalisés à la planche), dessinés par des artistes. Les exemples foisonnent. Citons encore les tensions autour de la classification des vitraux ${ }^{10}$ (certains peintres-verriers réclamant le statut de beaux-arts pour leurs œuvres) ou encore les conflits autour de la photographie après 1878, à l'heure de l'instantané au gélatino-bromure d'argent : «L'instantané [...] est accusé de tous les maux, mais d'abord comme une pratique inesthétique, la nouveauté technique et le progrès n'étant pas synonymes de beauté ${ }^{11}$. \" Plus généralement, dès 1855 , l'utopie technologique encyclopédique, héritière de l'union des arts mais aussi de l'ample mouvement de commercialisation du goût porté en Angleterre par la Society of Arts (instigatrice du Crystal Palace), vole en éclat : le " secours mutuel des arts » et « l'artialisation » des produits et des objets techniques, encensés par Maxime Du Camp, ne résistent pas aux critiques de Charles Baudelaire et d'Ernest Renan ${ }^{12}$. On prend donc la mesure de ce qui se joue pour l'architecture au début du $\mathrm{Xx}^{\mathrm{e}}$ siècle, et plus globalement pour la reconnaissance de l'esthétique industrielle.

On perçoit également, à rebours, l'originalité de la période qui précède, sur laquelle on est de mieux en mieux informé. Comme Fabienne Brugère le rappelle ici dans sa relecture de la Théorie des sentiments moraux, Adam Smith avait suggéré dès 1759 que l'utilité, lorsqu'elle se donnait à voir, était en elle-même porteuse d'une certaine beauté ${ }^{13}$. Et il donnait précisément l'exemple de la maison dont la commodité et la régularité manifestent de façon visible cette utilité : la belle maison, c'est la maison qui s'offre à nos yeux comme commode et confortable (nous ne sommes pas très loin du Grand Hippias). Impartial et doué d'empathie, le spectateur idéal dont la figure lui sert à fonder en raison sa théorie du jugement moral est susceptible, même si ce n'est

8. Hilaire-PÉrez (Liliane), «Les expositions universelles en France au XIX ${ }^{\mathrm{e}}$ siècle : lignes de tension et lignes d'horizon dans le champ technologique à l'ère de l'industrialisation », dans CARRÉ et al., 2012, p. 13-34.

9. BRIOT (Eugénie), «Un autel de Flore au temple de l'Industrie. La parfumerie française aux expositions universelles de 1889 et 1900 », dans CARRÉ et al., dir., 2012, p. 261-270.

10. LUNEAU (Jean-François), « Les peintres-verriers dans les expositions universelles : histoire d'un désamour », dans CARRÉ et al., dir., 2012, p. 247-259.

11. Perceval (Marion), «Un instantané des expositions universelles : les amateurs de photographies et les expositions de 1878 à 1900 », dans CARRÉ et al., dir., 2012, p. 363-374.

12. Caraion (Marta), "L'exposition universelle de 1855 : une réception biaisée », Wanlin (Nicolas), " "Du rêve condensé en fait". L'exposition universelle mise en vers, par delà matérialisme et idéalisme », 2012 ; JARRIGE (François), « Machines en mouvement. Les ambivalences du spectacle technique dans les expositions universelles du Second Empire ». Les trois articles sont parus dans CARré et al., dir, 2012, p. 49-60, 61-73 et 129-138.

13. « De la beauté que l'apparence de l'utilité confère à toutes les productions de l'art », SMITH, 1999 (cité par BRUGÈre, infra, p. 536). 
pas lui qui l'habite, d'apprécier la beauté d'une maison cossue sans pour autant en éprouver du ressentiment. En ce sens, on peut dire que la beauté des objets, en même temps qu'elle est la manifestation visible de leur utilité, a une véritable utilité sociale. Et cette utilité est même double puisqu'à cette fonction de régulation des passions dangereuses, on peut ajouter l'avantage social qu'en retire le riche propriétaire qui peut ainsi se faire connaître et admirer. Adam Smith s'adressait certes à des contemporains censément capables de percevoir la beauté inhérente à l'utilité du luxe, mais, eût-il connu les tombes princières de l'âge du Fer, où bijoux, vases et armes de prestige ont laissé l'éclatant témoignage du prix accordé à la puissance et à la gloire, qu'il s'en fût trouvé conforté dans ses convictions.

L'idée selon laquelle l'art ne joue aucun rôle fonctionnel, au contraire de la technique qui, elle, est utile est si communément admise que certains artistes en jouent. Il en est ainsi de Jean Tinguely dont les sculptures, faites de matériaux de récupération qu'il anime à l'aide de moteurs, n'ont d'autre rôle que de nous enchanter. Il s'amuse à détourner de leur usage des mécanismes familiers, et si ses machines fonctionnent merveilleusement bien, elles ne produisent rien. On a voulu y voir les vecteurs d'une critique de l'hyperconsommation, mais ils n'avaient cette possible utilité que dans la mesure où, comme machines, ils étaient inutiles. La machine inutile est devenu un genre sculptural en soi, et on en trouve même une étonnante illustration « brute » dans le musée de la Fabuloserie de Dicy (Yonne), due à un artiste connu seulement sous le nom de «Petit Pierre ». Point de message ici, sauf peut-être de la dérision mêlée de mélancolie. Et nous ne croyons pas qu'il y avait un message critique dans ce que Fred Vargas fait dire à l'un de ses personnages :

« Construire, mécaniser l'inutile. Je voulais faire un monument à la gloire de la mécanique! Et pour célébrer la beauté de la mécanique, je voulais que la machine ne serve à rien, son seul intérêt étant de marcher, de fonctionner, et qu'on puisse dire en la contemplant : "Ça marche !" Gloire au fonctionnement, et gloire au dérisoire et à l'inutile ! Gloire au levier qui pousse, à la roue qui tourne, au piston qui pistonne, au rouleau qui roule ! Et pour quoi faire ? Pour pousser, pour tourner, pour pistonner, pour rouler ${ }^{14}$ ! »

Cela nous ramène au XVIII ${ }^{\mathrm{e}}$ siècle, mais cette fois au monde de la bimbeloterie, du toyware anglais où nous entraîne Liliane Hilaire-Pérez. Objets inutiles mais conformes à ce qu'on attend d'eux - le jeu des apparences, le plaisir de l'artifice, le goût de la technicité -, ils témoignent d'une esthétique technique, d'une " techno-esthétique » au sens simondonien. En dissociant la technique de l'utilité, Simondon nous propose une autre vision de l'art, où toute œuvre d'art se structure à travers une individuation technique qui fait d'elle un objet inséparablement esthético-technique et technoesthétique ${ }^{15}$. "L L'œuvre est simultanément technique et esthétique, esthétique parce que technique, technique parce qu'esthétique ${ }^{16}$.»

14. VARGAS, 1996, p. 187.

15. DuHEM, 2012.

16. Voir l'article de Liliane HiLAIRE-PÉREz, publié infra, p. 495-524. 
Comme Fabienne Brugère, Liliane Hilaire-Pérez aborde la conception de l'esthétique chez Adam Smith. Et sans que les deux auteurs se soient concertées, leurs deux articles se font écho l'un l'autre. Alors que Fabienne Brugère, en philosophe, aborde la mise en place de cette « techno-esthétique » dans l'Angleterre au XVIII" siècle à partir du spectateur impartial imaginé par Adam Smith, Liliane Hilaire-Pérez, en historienne des techniques, voit dans cette mise en place une conception de l'esthétique où la beauté des objets est liée à leur aptitude à être utiles (aptness). Si les produits du toyware sont bien des frivolités " inutiles » et des emblèmes du jeu, ils promeuvent une certaine idée de l'efficacité des procédés et des moyens, de l'adaptation des objets aux usages pour lesquels ils ont été conçus. Et c'est précisément la parfaite adéquation de leur forme à leur destination qui fait leur valeur. De plus, si dépourvus qu'ils soient d'intérêt pratique, ils ont l'intérêt de témoigner de la variété des talents et des compétences qui ont permis leur fabrication. Mais cet intérêt n'est perceptible que pour nous et rétrospectivement, grâce à ce que nous savons de la suite de l'histoire des techniques.

Ainsi, un objet, quel qu'il soit, n'a de fonction et de valeur esthétique que celle que nous lui attribuons à un moment donné et son statut peut changer par le jeu de l'interaction avec un acteur. Un fétiche africain sera considéré en objet d'art ou en fétiche selon qu'il se trouve dans un musée ou dans son contexte d'origine ${ }^{17}$. Cet objet très utilitaire qu'est le fer à repasser de Marcel Duchamp devient un objet d'art du seul fait qu'il est placé dans la vitrine d'un musée ${ }^{18}$. Au point que Alfred Gell en vient à juger vain de s'interroger sur le beau et l'utile, sur l'esthétique et la fonction, une œuvre d'art n'existant que pour autant qu'elle est interprétée comme telle dans un monde de l'art historiquement déterminé ${ }^{19}$. Est-il sûr cependant que seuls les objets estampillés comme « artistiques » soient susceptibles d'un jugement esthétique ? Tout au plus peut-on dire que le jugement esthétique est parfois mis en suspens. Mais c'est moins alors les objets sur lesquels il s'exerce qui sont en cause, que les sujets qui, selon les circonstances, jugent opportun ou non de l'exercer.

\section{LISTE DES RÉFÉRENCES}

Bazin (Jean), 1996, « Des clous dans la Joconde », rééd. dans ID. Des clous dans la Joconde. L'anthropologie autrement, Toulouse, Anacharsis (Essais), 2008, p. 520-545.

BECQ (Annie), 1983, « La métaphore de la machine dans le discours esthétique de l'Âge classique », Revue des sciences humaines, p. 269-278.

Carré (Anne-Laure), Corcy (Marie-Sophie), Demeulenaere-Douyère (Christiane) et HilairePérez (Liliane), dir., 2012, Les Expositions universelles à Paris au XIX siècle. Techniques, publics, patrimoine, Paris, CNRS Éditions (Alpha).

DAmisch (Hubert), 1983, « De la manufacture comme œuvre d'art économique à l'œuvre d'art comme machine », Revue des sciences humaines, p. 307-320.

Deleule (Didier), 1997, « Préface. Adam Smith et la difficulté surmontée », dans Thierry, dir., 1997, p. 15-33.

17. BAZIN, 1996.

18. BAZIN, 1996, ici 2008, p. 525

19. GeLL, 1998. 
DÉmoris (René), 1983, « Chardin, la machine et l'oiseau », Revue des sciences humaines, p. 292-306.

Dubourg Glatigny (Pascal) et Vérin (Hélène), 2008, « La réduction en art, un phénomène culturel », dans ID., Réduire en art. La technologie de la Renaissance aux Lumières, Paris, Éditions de la Maison des sciences de l'Homme, p. 59-94.

Duhem (Ludovic), 2012, « Le milieu technique de l'art », dans Toulouse (Ivan), dir., Technique et création, Paris, L'Harmattan (Eurêka et $\mathrm{C}^{\mathrm{ie}}$ ), p. 14-37.

Gell (Alfred), 1998, Art and Agency. An Anthropological Theory, Oxford, Clarendon Press.

Hilaire-Pérez (Liliane), 2002, « Diderot's Views on Artists' and Inventors' Rights. Invention, Imitation and Reputation », British Journal for the History of Science, vol. 35, p. 129-150.

Millet (Audrey), 2011, «Penser la formation du dessinateur de fabrique à Tours : acteurs et réseaux (1776-1826) », dans Fonteneau (Virginie) et D’EnFERT (Renaud), éd., Espaces de l'enseignement scientifique et technique. Acteurs, savoirs, institutions, $X V I I^{e}-X X^{e}$ siècles, Paris, Hermann, p. 109-118.

Morvan (Frédéric), 2011, «L'école gratuite de dessin de Rouen ou la formation des techniciens au XVIII ${ }^{\mathrm{e}}$ siècle », doctorat de l'université Paris 8, Vincennes/Saint-Denis.

Reynolds (Joshua), 1797, Discourses on Art, rééd. Robert R. WARK, New Haven, Yale University Press, 1997.

Sсотт (Kate), 1999, « Chardin multiplié », dans Chardin, catalogue d'exposition, Paris, Réunion des musées nationaux, p. 61-73.

Sмiтн (Adam), 1795, « De la nature de l'imitation dans les arts que l'on appelle imitatifs », dans THIERRY, dir., 1997, p. 49-83.

Sмith (A.), 1999, Théorie des sentiments moraux, trad. fr. Michaël Bizıou, Claude Gautier et Jean-François Pradeau, Paris, Presses universitaires de France.

Thierry (Patrick), dir., 1997, Essais esthétiques, Paris, Vrin.

VARGAS (Fred), 1996, Un peu plus loin sur la droite, Paris, Viviane Hamy.

VÉRIN (Hélène), 1998, « La réduction en art et la science pratique au XVI ${ }^{\mathrm{e}}$ siècle », dans SALAIS (Robert), Chatel (Elisabeth) et Ribaud-Danset (Dorothée), éd., Institutions et conventions. La réflexivité de l'action économique, dossier thématique de Raisons Pratiques, $\mathrm{n}^{\circ} 9$, p. 121-144.

Ce numéro est dédié à la mémoire de François Sigaut (10 novembre 1940-2 novembre 2012), directeur d'études à l'EHESS, dont nous avons appris la disparition au moment de la mise sous presse. Spécialiste de la pensée technique et défenseur de la technologie comme science de l'homme, il venait de publier Comment Homo devint faber, aux éditions du CNRS. Son parcours, ses travaux et ses publications feront l'objet d'un article dans un prochain numéro de la Revue. 